\title{
Salvage treatment with apatinib for advanced non-small-cell lung cancer
}

This article was published in the following Dove Press journal:

OncoTargets and Therapy

23 March 2017

Number of times this article has been viewed

\author{
Zhengbo Song ${ }^{1,2}$ \\ Xinmin $Y u^{1,2}$ \\ Guangyuan Lou ${ }^{1,2}$ \\ Xun Shi, \\ Yiping Zhang ${ }^{1,2}$ \\ 'Department of Chemotherapy, \\ Zhejiang Cancer Hospital, ${ }^{2}$ Key \\ Laboratory Diagnosis and Treatment \\ Technology on Thoracic Oncology, \\ Zhejiang province, Hangzhou, People's \\ Republic of China
}

\begin{abstract}
Objective: No definitive chemotherapeutic regimen has been established in patients with non-small-cell lung cancer (NSCLC) who failed second- or third-line treatment. The aim of this study was to evaluate apatinib, a VEGFR-2 inhibitor, in advanced NSCLC as salvage treatment.
\end{abstract}

Methods: We evaluated the efficacy and toxicity of apatinib in patients with previously treated advanced NSCLC from 2014 to 2015 in Zhejiang Cancer Hospital. Survival analysis was performed by the Kaplan-Meier method.

Results: Forty-two patients were included in the present study. Four patients achieved partial response, and 22 achieved stable disease, representing a response rate of $9.5 \%$ and a disease control rate of $61.9 \%$. Median progression-free survival and overall survival were 4.2 and 6.0 months, respectively. The toxicities associated with apatinib were generally acceptable with a total grade $3 / 4$ toxicity of $50 \%$.

Conclusion: Apatinib appears to have some activity against advanced NSCLC when utilized as salvage treatment.

Keywords: non-small-cell lung cancer, apatinib, VEGF, efficacy

\section{Introduction}

Lung cancer is one of the leading causes of cancer-related death worldwide. ${ }^{1}$ For patients with advanced non-small-cell lung cancer (NSCLC), chemotherapy and targeted therapy can prolong survival time and are recommended as standard treatment in advanced NSCLC. ${ }^{2-5}$ However, there is no definitive therapeutic regimen in patients who failed second-line/third-line treatment. Many patients receive further chemotherapy in practice. ${ }^{6}$

Treatment by inhibition of VEGFs has been shown to be effective in many solid tumors. ${ }^{7-9}$ Bevacizumab, an anti-VEGF monoclonal antibody, has been shown to significantly improve overall survival (OS) in advanced NSCLC in several prospective studies. ${ }^{10,11}$ Preclinical and clinical experiments indicated that apatinib, a novel VEGFR inhibitor, has potential as a therapeutic agent for carcinomas. ${ }^{12}$ Apatinib is a small-molecule VEGFR-2 tyrosine kinase inhibitor that improved progression-free survival (PFS) and OS in heavily pretreated patients with metastatic gastric cancer. ${ }^{13,14}$ Preliminary results of an apatinib study presented at the ASCO meeting in 2012 (abstract 7548) reported efficacy in NSCLC. ${ }^{15}$ However, no clinical studies with detailed data have investigated the efficacy of apatinib in NSCLC as salvage treatment.

In the present study, we conducted a retrospective evaluation of the efficacy and toxicity of apatinib after failure of prior treatment in advanced NSCLC. 


\section{Methods}

\section{Patient eligibility}

Patients with advanced NSCLC who received apatinib as third-line or further treatment between 2014 and 2015 were recruited. Inclusion criteria were 1) histological or cytological diagnosis of NSCLC according to histopathological criteria (World Health Organization, 2004); 2) lack of local treatment such as radiotherapy or interventional therapy during the duration of apatinib therapy; and 3) recurrence or metastases identified in chest, abdominal, or brain computed tomography (CT) scans and/or bone scans. The study was approved by the institutional review board of Zhejiang Cancer Hospital.

\section{Treatment methods}

Apatinib was administered at a dose of $500 \mathrm{mg}$ once daily. One treatment cycle was 28 days long. One dose reduction (to $250 \mathrm{mg}$ ) for drug-related toxicity was allowed.

\section{Responses and toxicity}

Tumor responses were assessed every two cycles or were evaluated early when significant signs of progression appeared. Objective tumor responses were assessed according to the Response Evaluation Criteria in Solid Tumors (RECIST 1.1). Objective tumor responses included complete response (CR), partial response (PR), stable disease (SD), and progressive disease (PD). The disease control rate (DCR) was defined as the addition of objective response and stabilization rates $(\mathrm{CR}+\mathrm{PR}+\mathrm{SD})$. Toxicities were evaluated according to the National Cancer Institute Common Toxicity Criteria version 4.0 (CTC4.0).

\section{Follow-up}

All the patients who were evaluated for tumor response had PFS and OS. PFS encompassed the time from the first day of apatinib to documented progression or death from any cause. OS was defined as the time from the first day of apatinib treatment to death or last follow-up. The median follow-up period was 12.5 months (3.0-23). Follow-up extended till April 30, 2016.

\section{Statistical analysis}

Survival analysis was conducted by the Kaplan-Meier analysis and comparison by the log-rank test. The survival curves were calculated according to the Kaplan-Meier method. Statistical analysis was performed using SPSS version 18.0 (SPSS Inc., Chicago, IL, USA). $P<0.05$ was regarded as statistically significant.

\section{Results \\ Patients' characteristics}

A total of 42 patients were included; of these, 12 had squamous cell carcinoma and 30 had adenocarcinoma. Seventeen had smoking history and 25 were never-smokers. Twenty-four patients were male and 18 were female, and they had a median age of 56.5 years. Tumor samples for gene mutation analysis were available for 18 patients. EGFR mutations were identified in seven patients (five with deletion in exon 19 and two with L858R in exon 21). All patients received palliative chemotherapy and 29 had targeted therapy in prior treatment. Twenty-two patients had been found to have PR (responder) status and $20 \mathrm{SD}$ or PD (nonresponder) status during prior treatment. Nineteen patients received apatinib as third-line therapy and 23 as further-line treatment. Twenty-five patients had performance status (PS) of $0-1$ and 17 had PS of 2. The patient characteristics are listed in Table 1.

\section{Clinical efficacy}

Response data for apatinib are as follows: $\mathrm{CR}(\mathrm{n}=0), \mathrm{PR}$ $(\mathrm{n}=4), \mathrm{SD}(\mathrm{n}=22)$, and PD $(\mathrm{n}=16)$. The ORR and DCR were 9.5 and $61.9 \%$, respectively. The median PFS was 4.2 months (95\% CI, 1.0-9.5; Figure 1). The median OS was 6.0 months

Table I Clinical characteristics of the study population $(n=42)$

\begin{tabular}{ll}
\hline Variables & Number (\%) \\
\hline Gender & \\
Male & $24(57.1)$ \\
Female & $18(42.9)$ \\
PS & \\
$0-I$ & $25(59.5)$ \\
2 & $17(40.5)$ \\
Age, years & \\
Median (range) & $56.5(42-75)$ \\
$>60$ & $10(23.8)$ \\
$\leq 60$ & $32(76.2)$ \\
Smoking history & \\
Yes & $17(40.5)$ \\
No & $25(59.5)$ \\
Histology & \\
Adenocarcinoma & $30(71.4)$ \\
Squamous cell carcinoma & $12(28.6)$ \\
EGFR mutation & \\
Yes & $7(16.7)$ \\
No & $11(26.2)$ \\
Unknown & $24(57.1)$ \\
Line of apatinib therapy & \\
Third line & $19(45.2)$ \\
Further line & $23(54.8)$ \\
Targeted treatment in prior therapy & \\
Yes & $29(69.0)$ \\
No & $13(31.0)$ \\
\hline
\end{tabular}

Abbreviation: PS, performance status. 


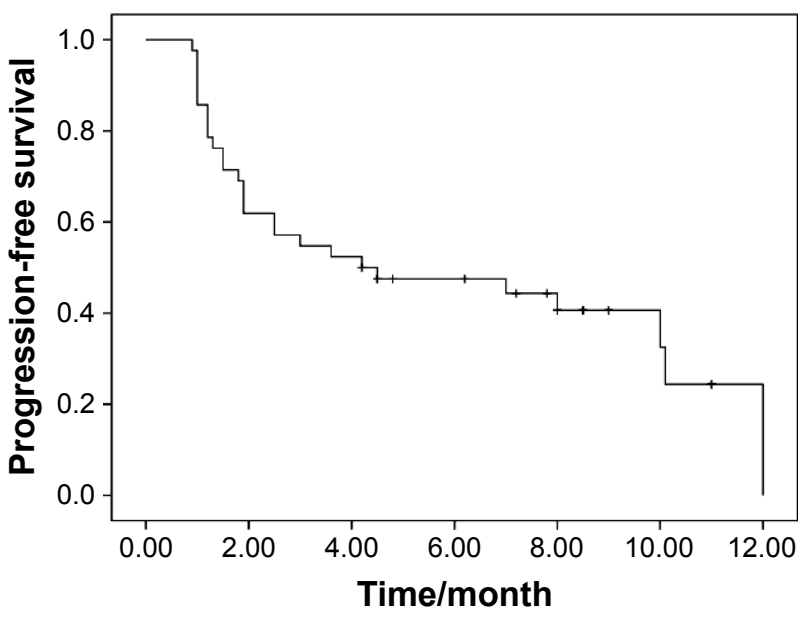

Figure I Kaplan-Meier estimates of progression-free survival of apatinib treatment.

(95\% CI, 3.9-8.0; Figure 2). There was no significant association of PFS with gender $(P=0.06)$, age $(P=0.13)$, line of therapy $(P=0.43)$, pathological subtype $(P=0.36)$, smoking history $(P=0.34)$, and PS $(P=0.07)$. The EGFR mutation status and response to prior treatment were not found to be statistically associated with PFS after apatinib treatment $(P=0.25$ and 0.42 , respectively).

Interestingly, EGFR-TKI treatment was administered to two patients after apatinib, and the PFS were 11.0 and 9.0 months, respectively. The details of univariate analysis are shown in Table 2.

\section{Toxicity evaluation}

All patients were assessed for toxicity. The rate of grade 3/4 toxicity was $50 \%$. Grade 4 toxicity was not observed in all the patients. Two patients discontinued the apatinib treatment because they developed deteriorated hepatic function

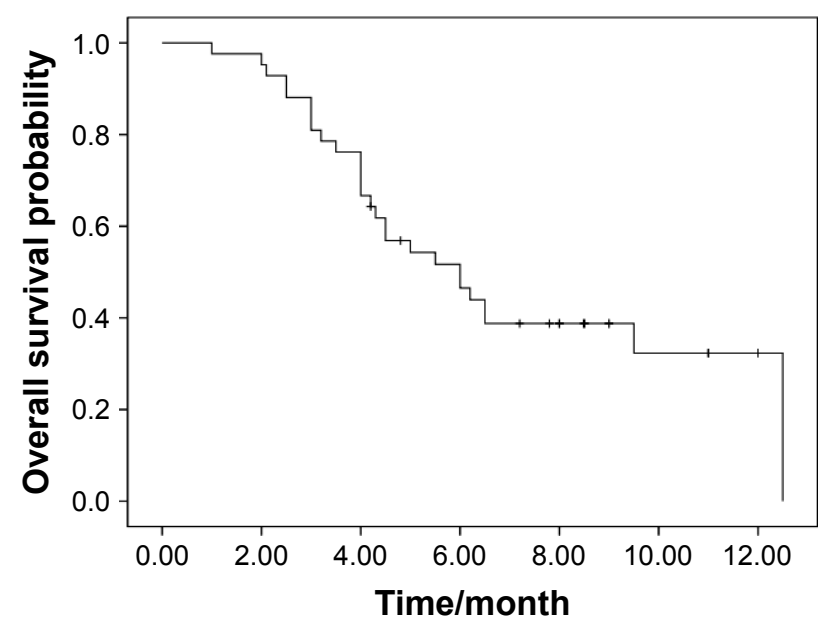

Figure 2 Kaplan-Meier estimates of overall survival of apatinib treatment. and hand-foot syndrome. Four patients required dosage reductions. The common grade $3 / 4$ adverse events were hand-foot syndrome $(\mathrm{n}=6)$, hypertension $(\mathrm{n}=4)$, proteinuria $(\mathrm{n}=3)$, hepatic injury $(\mathrm{n}=3)$, fatigue $(\mathrm{n}=2)$, and nausea/vomiting ( $\mathrm{n}=2$; Table 3$)$.

\section{Discussion}

To our knowledge, this is the first study to assess whether apatinib as salvage chemotherapy confers clinical benefit in patients with advanced NSCLC. The results demonstrated that apatinib had efficacy against advanced NSCLC in laterline treatment.

Platinum-based chemotherapy is widely used in routine NSCLC treatment. For patients with some gene mutations, targeted therapy has been identified that can prolong survival and preserve quality of life..$^{3-5}$ The present results are consistent with the use of targeted therapy for second-line therapy when given to $40 \%-60 \%$ of patients and to $20 \%-30 \%$ of patients for third-line or further therapy for decreased PS and lower toxicity. ${ }^{16,17}$ Regimens with low toxicity and good efficacy are not available in China for patients in these clinical settings. For patients who have failed third-line treatment, no standard treatment has been recommended.

Apatinib is a novel small-molecule protein tyrosine kinase (PTK) inhibitor that selectively targets VEGFR-2, which binds all VEGF-A isoforms, VEGF-C, and VEGF-D. The binding of VEGF-A to VEGFR-2 can induce a cascade of signaling pathways, eventually causing cellular proliferation and endothelial cell survival. ${ }^{18,19}$ Several studies have confirmed that blocking VEGFR-2 was a promising therapy for inhibiting angiogenesis. ${ }^{20,21}$ Apatinib, the first oral VEGFR-2 inhibitor, has been shown to have survival benefit compared with placebo in advanced gastric cancer in a Phase III trial (6.5 vs 4.7 months, $P=0.0149$ ) ${ }^{14}$ and has been approved in China for the treatment of patients with advanced gastric cancer refractory to two or more lines of prior chemotherapy. Apatinib was also reported to be an effective salvage therapy agent in advanced breast carcinoma patients who failed standard treatment (PFS: 3.3 months, OS: 10.7 months). ${ }^{22}$ Two cases treated with apatinib have been reported in NSCLC patients with PFS of 4.6 and 6 months. ${ }^{23}$

In the present study, the DCR and ORR were 61.9 and $9.5 \%$, which indicates superior efficacy as second-line chemotherapy over docetaxel in NSCLC. ${ }^{24}$ Of note, more than one-third of patients in the present study had PS of 2, which may influence the efficacy of apatinib. Bevacizumab, another antiangiogenesis drug, is not approved for the treatment of squamous cell carcinoma because of hemorrhage. ${ }^{24}$ In the 
Table 2 Univariate analysis of PFS and OS

\begin{tabular}{|c|c|c|c|c|c|c|}
\hline & PFS & $95 \% \mathrm{Cl}$ & $P$-value & OS & $95 \% \mathrm{Cl}$ & $P$-value \\
\hline Gender & & & 0.06 & & & 0.12 \\
\hline Male & 2.5 & $1.4-3.6$ & & 5.2 & $3.2-5.7$ & \\
\hline Female & 10.0 & $4.7-12.3$ & & 10.7 & $5.1-14.1$ & \\
\hline Age, years & & & 0.13 & & & 0.21 \\
\hline$>60$ & 7.0 & $1.2-12.3$ & & 4.2 & I.5-5.5 & \\
\hline$\leq 60$ & 1.2 & $0.4-2.0$ & & 6.5 & $4.2-7.9$ & \\
\hline PS & & & 0.07 & & & 0.09 \\
\hline $0-1$ & 10.0 & $1.2-13.5$ & & 8.5 & $5.4-14.2$ & \\
\hline 2 & 3.0 & $0.5-5.5$ & & 4.5 & $3.0-6.8$ & \\
\hline Line of therapy & & & 0.43 & & & 0.31 \\
\hline Third-line & 2.5 & $0.0-8.4$ & & 7.2 & $3.8-10.5$ & \\
\hline Further-line & 8.0 & $0.1-12.9$ & & 5.5 & $4.0-7.7$ & \\
\hline Smoking history & & & 0.34 & & & 0.47 \\
\hline Yes & 2.5 & $1.5-3.5$ & & 5.5 & $3.0-8.5$ & \\
\hline No & 8.0 & $5.2-10.8$ & & 7.8 & $6.0-10.5$ & \\
\hline Histology & & & 0.36 & & & 0.17 \\
\hline Adenocarcinoma & 7.0 & $0.0-12.6$ & & 6.5 & $0.3-12.7$ & \\
\hline Squamous cell & 1.9 & $0.0-5.0$ & & 4.3 & $2.1-8.5$ & \\
\hline EGFR status & & & 0.25 & & & 0.87 \\
\hline Mutation & 4.4 & $0.6-4.8$ & & 7.0 & $5.0-9.5$ & \\
\hline Wild-type & 4.0 & $1.0-6.0$ & & 5.5 & $4.0-6.5$ & \\
\hline Prior treatment efficacy & & & 0.42 & & & 0.55 \\
\hline Responder & 4.6 & $1.2-6.2$ & & 7.5 & $3.5-10.0$ & \\
\hline Nonresponder & 3.5 & $0.0-5.0$ & & 5.0 & $2.5-9.0$ & \\
\hline
\end{tabular}

Abbreviations: PFS, progression-free survival; OS, overall survival; PS, performance status.

present study, 12 patients with squamous cell carcinoma were treated, and no hemorrhage occurred, suggesting safety of this oral inhibitor. Combination of an antiangiogenesis drug and EGFR-TKIs showed a promising therapy in NSCLC. ${ }^{25}$ Interestingly, two of our patients continued on a combination of EGFR-TKI and apatinib after progression on prior EGFR-TKI. Better efficacy was found in both these patients. This combination treatment may prove to be effective therapy after wider validation.

The most common adverse events with apatinib treatment were skin reactions of the hands and feet ("hand-foot syndrome"), proteinuria, and hypertension with total grade $3 / 4$ adverse events of $\sim 60 \%$ in gastric carcinoma. ${ }^{13,14}$ More than $20 \%$ of patients required dose modifications after being started

Table 3 Main grade 3/4 toxicities of apatinib treatment

\begin{tabular}{lll}
\hline Toxicity & Grades 3-4 & Percentage \\
\hline Hand-foot syndrome & 6 & 14.3 \\
Hypertension & 4 & 9.5 \\
Proteinuria & 3 & 7.1 \\
Hepatic injure & 3 & 7.1 \\
Fatigue & 2 & 4.8 \\
Nausea/vomiting & 2 & 4.8 \\
Febrile neutropenia & $\mathrm{I}$ & 2.4 \\
Anemia & $\mathrm{I}$ & 2.4 \\
\hline
\end{tabular}

on the recommended dose of apatinib ( $850 \mathrm{mg}$ daily) treatment in a Phase 3 study. ${ }^{14}$ In the study of Hu et al, ${ }^{22}$ where the recommended dose was $500 \mathrm{mg}$ daily, the rate of grade 3/4 toxicity was significantly decreased but the efficacy was similar to that of the high-dose regimen in breast carcinoma. In the present study, we used the $500 \mathrm{mg}$ recommended dose in our patients, and the results showed that half of these patients developed grade 3 toxicities, but none showed grade 4 toxicities.

The major limitation of the present study is its retrospective nature and small number of patients. There was also some heterogeneity in the study population, with different histology and clinical characteristics occurring. Finally, the dose of $500 \mathrm{mg}$ was used in the present study, which should be confirmed in future trials. Two prospective clinical studies that are ongoing in China (NCT01287962 and NCT01270386) currently will provide further data on the efficacy of apatinib in patients with advanced NSCLC.

\section{Conclusion}

In summary, the present results suggest that apatinib is an effective regimen for salvage treatment of advanced NSCLC, but further prospective studies with larger numbers of patients are required to fully establish the efficacy and safety of this treatment. 


\section{Disclosure}

The authors report no conflicts of interest in this work.

\section{References}

1. Chen W, Zheng R, Baade PD, et al. Cancer statistics in China, 2015. CA Cancer J Clin. 2016;66(2):115-132.

2. Mitsudomi T, Morita S, Yatabe Y, et al; West Japan Oncology Group. Gefitinib versus cisplatin plus docetaxel in patients with non-smallcell lung cancer harbouring mutations of the epidermal growth factor receptor (WJTOG3405): an open label, randomised phase 3 trial. Lancet Oncol. 2010;11(2):121-128.

3. Zhou C, Wu YL, Chen G, et al. Erlotinib versus chemotherapy as first-line treatment for patients with advanced EGFR mutation-positive non-small-cell lung cancer (OPTIMAL, CTONG-0802): a multicentre, open-label, randomised, phase 3 study. Lancet Oncol. 2011;12(8): 735-742.

4. Mok TS, Wu YL, Thongprasert S, et al. Gefitinib or carboplatin paclitaxel in pulmonary adenocarcinoma. $N$ Engl J Med. 2009;361:947-957.

5. Shaw AT, Kim DW, Nakagawa K, et al. Crizotinib versus chemotherapy in advanced ALK-positive lung cancer. $N$ Engl $J$ Med. 2013;368(25):2385-2394.

6. Song Z, Zhang Y. Retreatment with pemetrexed chemotherapy in advanced non-small cell lung cancer patient. $J$ Thorac Dis. 2014;6: 856-860.

7. Garon EB, Ciuleanu TE, Arrieta O, et al. Ramucirumab plus docetaxel versus placebo plus docetaxel for second-line treatment of stage IV non-small-cell lung cancer after disease progression on platinumbased therapy (REVEL): a multicentre, double-blind, randomised phase 3 trial. Lancet. 2014;384(9944):665-673.

8. Hall RD, Le TM, Haggstrom DE, Gentzler RD. Angiogenesis inhibition as a therapeutic strategy in non-small cell lung cancer (NSCLC). Transl Lung Cancer Res. 2015;4:515-523.

9. Hanrahan EO, Heymach JV. Vascular endothelial growth factor receptor tyrosine kinase inhibitors vandetanib (ZD6474) and AZD2171 in lung cancer. Clin Cancer Res. 2007;13(15 pt 2):s4617-s4622.

10. Sandler A, Gray R, Perry MC, et al. Paclitaxel-carboplatin alone or with bevacizumab for non-small-cell lung cancer. $N$ Engl $J$ Med. 2006;355(24):2542-2550.

11. Reck M, von Pawel J, Zatloukal P, et al. Phase III trial of cisplatin plus gemcitabine with either placebo or bevacizumab as first-line therapy for nonsquamous non-small-cell lung cancer: AVAil. J Clin Oncol. 2009;27(8):1227-1234.

12. Scott AJ, Messersmith WA, Jimeno A. Apatinib: a promising oral antiangiogenic agent in the treatment of multiple solid tumors. Drugs Today (Barc). 2015;51(4):223-229.
13. Li J, Qin S, Xu J, et al. Apatinib for chemotherapy-refractory advanced metastatic gastric cancer: results from a randomized, placebo-controlled, parallel-arm, phase II trial. J Clin Oncol. 2013;31(26):3219-3225.

14. Li J, Qin S, Xu J, et al. Randomized, double-blind, placebo-controlled phase III trial of apatinib in patients with chemotherapy-refractory advanced or metastatic adenocarcinoma of the stomach or gastroesophageal junction. J Clin Oncol. 2016;34(13):1448-1454.

15. Zhang K, Shi M, Huang C, et al. A phase II, multicenter, placebocontrolled trial of apatinib in patients with advanced nonsquamous non-small cell lung cancer (NSCLC) after two previous treatment regimens. J Clin Oncol. 2010;15(suppl):7548.

16. Sun JM, Lee KW, Kim JH, et al. Efficacy and toxicity of pemetrexed as a third-line treatment for non-small cell lung cancer. Jpn J Clin Oncol. 2008;39:27-32.

17. Girard N, Jacoulet P, Gainet M, et al. Third-line chemotherapy in advanced non-small cell lung cancer: identifying the candidates for routine practice. J Thorac Oncol. 2009;4(12):1544-1549.

18. Ding J, Chen X, Gao Z, et al. Metabolism and pharmacokinetics of novel selective vascular endothelial growth factor receptor-2 inhibitor apatinib in humans. Drug Metab Dispos. 2013;41:1195-1210.

19. Aziz MA, Serya RA, Lasheen DS, et al. Discovery of potent VEGFR-2 inhibitors based on furopyrimidine and thienopyrimidne scaffolds as cancer targeting agents. Sci Rep. 2016;6:24460.

20. Zhang HQ, Gong FH, Li CG, et al. Design and discovery of 4-anilinoquinazoline-acylamino derivatives as EGFR and VEGFR-2 dual TK inhibitors. Eur J Med Chem. 2016;109:371-379.

21. Ghavamipour F, Shahangian SS, Sajedi RH, Arab SS, Mansouri K, Aghamaali MR. Development of a highly-potent anti-angiogenic VEGF8-109 heterodimer by directed blocking of its VEGFR-2 binding site. FEBS J. 2014;281(19):4479-4494.

22. Hu X, Zhang J, Xu B, et al. Multicenter phase II study of apatinib, a novel VEGFR inhibitor in heavily pretreated patients with metastatic triple-negative breast cancer. Int J Cancer. 2014;135(8):1961-1969.

23. Ding L, Li QJ, You KY, Jiang ZM, Yao HR. The use of apatinib in treating nonsmall-cell lung cancer: case report and review of literature. Medicine (Baltimore). 2016;95(20):e3598.

24. Shepherd FA, Dancey J, Ramlau R, et al. Prospective randomized trial of docetaxel versus best supportive care in patients with non-small-cell lung cancer previously treated with platinum-based chemotherapy. J Clin Oncol. 2000;18(10):2095-2103.

25. Seto T, Kato T, Nishio M, et al. Erlotinib alone or with bevacizumab as first-line therapy in patients with advanced non-squamous non-smallcell lung cancer harbouring EGFR mutations (JO25567): an open-label, randomised, multicentre, phase 2 study. Lancet Oncol. 2014;15(11): $1236-1244$.
OncoTargets and Therapy

\section{Publish your work in this journal}

OncoTargets and Therapy is an international, peer-reviewed, open access journal focusing on the pathological basis of all cancers, potential targets for therapy and treatment protocols employed to improve the management of cancer patients. The journal also focuses on the impact of management programs and new therapeutic agents and protocols on

\section{Dovepress}

patient perspectives such as quality of life, adherence and satisfaction. The manuscript management system is completely online and includes a very quick and fair peer-review system, which is all easy to use. Visit http://www.dovepress.com/testimonials.php to read real quotes from published authors. 